\title{
Effect of sex and sex steroids on brown adipose tissue heat production in humans
}

\author{
John-Paul Fuller-Jackson' ${ }^{1}$, Aimee L Dordevic ${ }^{2}$, lain J Clarke ${ }^{3}$ and Belinda A Henry 1 \\ ${ }^{1}$ Metabolism, Diabetes and Obesity Program, Monash Biomedicine Discovery Institute, Department of Physiology, \\ Monash University, Clayton, Victoria, Australia, ${ }^{2}$ Department of Nutrition, Dietetics and Food, Monash University, \\ Victoria, Australia, and ${ }^{3}$ Neuroscience Program, Biomedicine Discovery Institute, Department of Physiology, Monash \\ University, Victoria, Australia
}

Correspondence should be addressed to B A Henry

Email

belinda.henry@monash.edu

\begin{abstract}
Objective: Retrospective studies suggest that women have more active brown adipose tissue (BAT) than men, but little is known of the effect of fluctuating sex steroids across the menstrual cycle on thermogenesis in women.

Design: To characterise the effects of sex and sex steroids on BAT activity we recruited healthy weight men $(n=14)$ and women at two stages of the menstrual cycle (luteal, $n=9$; follicular, $n=11$ ).

Methods: Infrared thermography measured supraclavicular temperature to index BAT thermogenesis in response to both cold (immersion of one hand in water at $15^{\circ} \mathrm{C}$ ) and meal (Ensure, $10 \mathrm{kcal} / \mathrm{kg}$ body weight) stimuli. Results: Adaptive BAT temperature responses were greater $(P<0.05)$ in women than men, irrespective of stage of menstrual cycle. Whereas during cold exposure, the increase in BAT temperature was abrogated $(P<0.05)$ in women during follicular phase compared to men and women during luteal phase. Plasma concentrations of progesterone, $17 \beta$-estradiol, testosterone and cortisol were measured. Regression analyses demonstrated that baseline BAT temperature was positively correlated $(P<0.05)$ with progesterone levels, but was inversely associated $(P<0.05)$ with cortisol concentration. Both cold- and meal-induced changes in BAT temperature mildly correlated $(P=0.07 ; P<0.05)$ with $17 \beta$-estradiol levels, but not with testosterone concentrations.

Conclusions: Baseline supraclavicular temperature is elevated in women during the luteal phase of the menstrual cycle, which correlated with elevated progesterone concentrations. Women exhibited greater thermogenic responses than men, irrespective of the state of the menstrual cycle, which was associated with plasma levels of $17 \beta$-estradiol. We conclude that sex steroids may regulate BAT thermogenesis in healthy adults.
\end{abstract}

\section{Introduction}

Thermogenesis contributes to approximately $10 \%$ of total energy expenditure in lean individuals (1). In humans, brown and beige adipose tissue (BAT) as well as skeletal muscle contribute to total thermogenic capacity (2). In adult humans, BAT is located in the supraclavicular, neck, epicardial, peri-renal and para-spinal adipose depots (3). In the neck and supraclavicular regions, adipose tissue contains a heterogeneous population of white, brown and beige adipocytes (4), with the latter two cell types being capable of thermogenesis. Beige adipocytes are derived from the white adipocyte population (5), either being formed from preadipocytes (6) or being recruited by transdifferentiation of white adipocytes (7). In humans chronic activation of BAT via repeated cold exposure increases energy expenditure and decreases adiposity (8). Furthermore, cold-induced BAT activation also increases glucose and triglyceride clearance and improves insulin sensitivity in adults $(9,10)$. Despite this, it remains contentious as to the precise physiological role and importance of BAT in metabolism and energy expenditure in humans (11). 
A number of studies have suggested that the regulation of BAT may be sexually dimorphic, yet, few studies have addressed this directly. Retrospective PET-CT studies suggest a greater prevalence of active BAT in women than in men (12) and data from animal models show that estrogen treatment increases thermogenesis in both BAT and skeletal muscle $(13,14)$. Despite this, a recent prospective study using PET-CT imaging suggested that women had a lower supraclavicular BAT volume than men, albeit ${ }^{18} \mathrm{~F}$-FDG uptake being comparable in men and women suggesting that overall supraclavicular BAT activity is similar, irrespective of sex (15). The study of Fletcher et al., however, identified a BAT depot in the superficial dorsocervical region that was more prevalent in women than men (15). Therefore, there appears to be sexual dimorphism in the distribution and activity of BAT, but whether this tissue exerts greater metabolic action in men or women remains to be elucidated. Furthermore, it is important to highlight a recent study in mice, which demonstrated that $\beta 3$ adrenoceptor-induced ${ }^{18} \mathrm{~F}$ FDG uptake was independent of uncoupling protein 1 (UCP1); CL-316,243 treatment increased glucose uptake to an equivalent degree in Ucp1 WT and knockout mice (16). This highlights the importance of validating new techniques to measure BAT activity in humans.

In addition to the sex differences, body temperature fluctuates across the menstrual cycle in women being higher during the luteal phase (17) when progesterone levels are elevated. Furthermore, women have a greater tolerance to cold during the luteal phase, which is evidenced by increased latency to the onset of shivering during cold exposure compared to women during the follicular phase (18). This may be due, in part, to the changes in vasomotor control of blood flow to the skin (18), but it remains possible that alterations in thermogenesis contribute to changes in body temperature in women during different phases of the menstrual cycle. Indeed, the previous study of Fletcher et al. (15) examined women during the follicular phase only. In vitro studies have suggested that progesterone may act directly at brown adipocytes, whereby progesterone treatment increases Ucp1 mRNA expression (19) and enhances noradrenalineinduced lipolysis in cultured brown adipocytes (20), thus emphasising the possible importance of the stage of cycle. The present study recruited healthy young adults and characterised both cold- and meal-induced changes in BAT temperature in men and women. We hypothesized that thermogenesis induced by both cold and meal-stimulus is greater in women than men. We hypothesized further that baseline, cold- and diet-induced changes in supraclavicular temperature will be greater in women during the luteal phase of the menstrual cycle than either men or women during the follicular phase. We predicted that such changes would be correlated with plasma sex steroid concentrations.

\section{Methods}

\section{Participants}

Fourteen male and 20 female healthy, young participants between the ages of 18-40 years old were recruited for this study. All participants had no history of cardiovascular disease, diabetes and were all non-smokers, not pregnant or lactating and were not taking any form of hormonal contraceptive. Ethical approval was obtained from the Monash University Human Research Ethics Committee for the study, which adhered to the requirements of the National Statement on Ethical Conduct in Human Research but also the Declaration of Helsinki, Seventh Revision, 2013. Written informed consent was obtained from each participant prior to the commencement of study.

\section{Study design}

The overall study design is outlined in Fig. 1A. Infrared thermography was used to measure temperature changes in the supraclavicular and manubrium regions to provide assessment of altered heat production in both BAT-positive and BAT-negative sites, respectively, as discussed in detail below. Changes in heat production were measured in response to both cold and meal stimuli (see below). Female participants were required to record their onset of menstruation and measure daily basal oral temperature for 4 weeks to provide indication of the stage of menstrual cycle and the studies were performed during either the follicular or the luteal phases of the menstrual cycle. To confirm the stage of menstrual cycle, blood samples were collected and used to measure plasma progesterone and $17 \beta$-estradiol concentrations.

Infrared thermal imaging was performed during the morning between 07:00-12:00 h (Fig. 1B). Participants arrived after fasting overnight and were rested for $30 \mathrm{~min}$ prior to any experimentation. During this rest period, a single catheter (22G Introcan Safety IV Catheter, B. Braun Australia, VIC, Australia) was inserted into the antecubital vein and used for the collection of the baseline blood sample $(10 \mathrm{~mL})$. Blood samples were collected into plasma separation tubes (Becton Dickinson Pty Ltd, VIC, Australia) and plasma was harvested via centrifugation at 
A
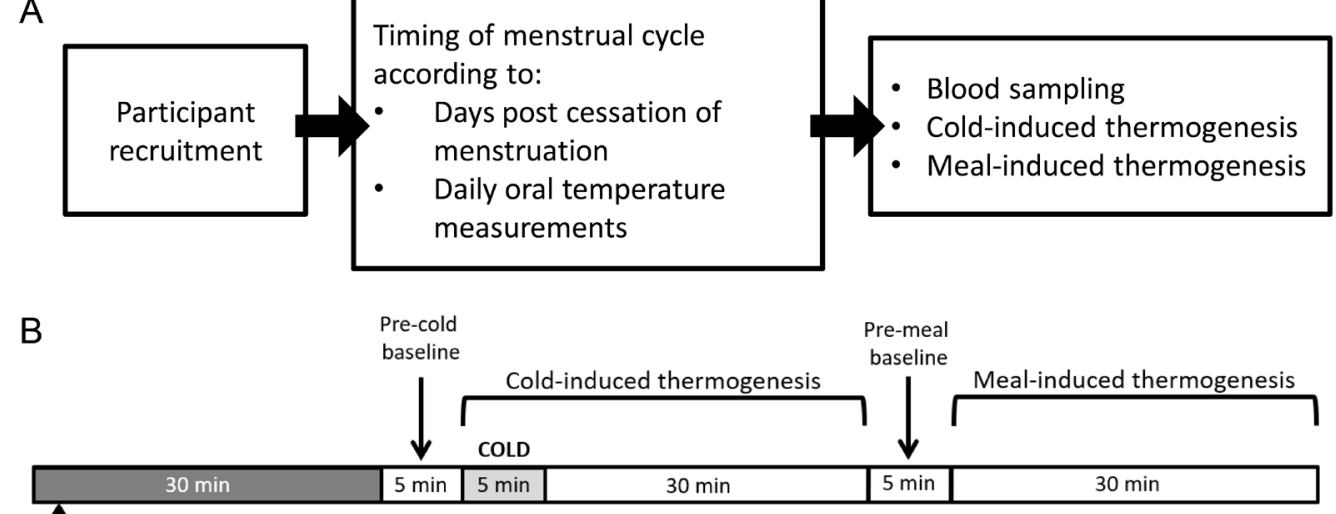

Baseline blood sample

C

Baseline
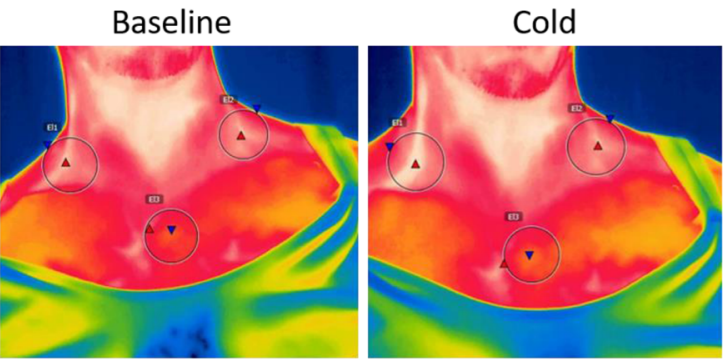

Meal

Male

Female Follicular phase
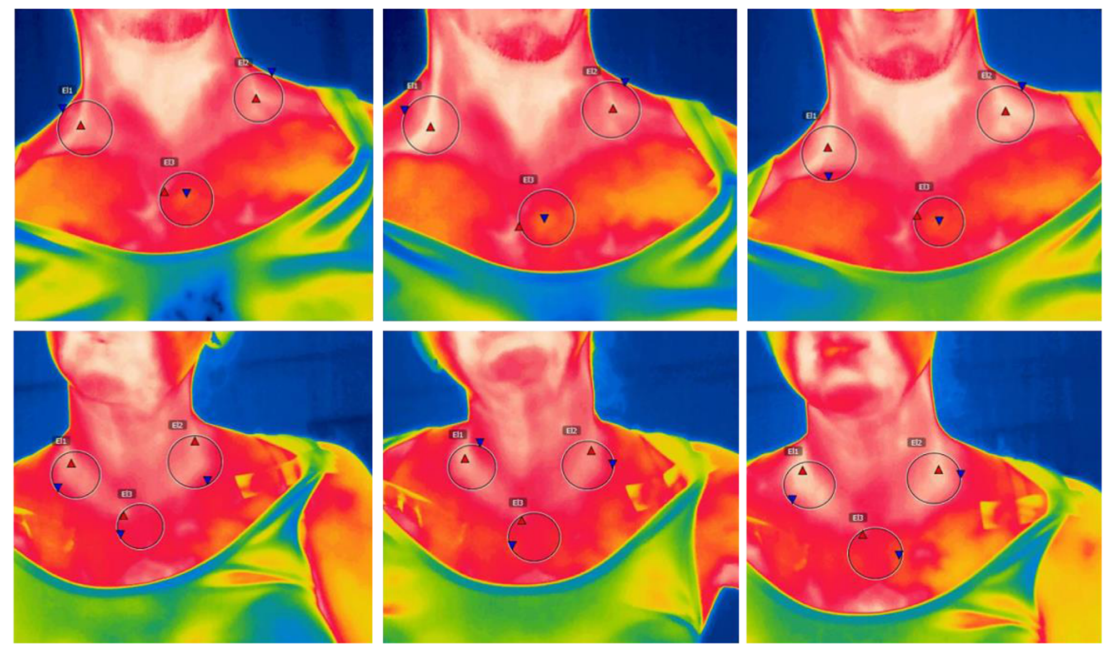

$35.0^{\circ} \mathrm{C}$

Female


Luteal phase

\section{Figure 1}

Experimental design for thermal imaging studies. Prior to experimentation, women were asked to maintain a menstrual diary recording the first and last day menstruation. In addition, women recorded daily basal body temperature using an oral thermometer (Panel A). This information was then used to estimate the stage menstrual cycle prior to thermography recordings (Panel B). On the day of experimentation, upon arrival, a cannula was inserted into the ante-cubital vein and a baseline blood sample was collected and used to measure plasma levels of progesterone, 17 $\beta$-estradiol and testosterone. Participants rested for 30 min prior to temperature recordings. Cold exposure was elicited by having participants immerse one hand in a bucket of water at $15^{\circ} \mathrm{C}$ for $5 \mathrm{~min}$, after which participants consumed a standardised meal (10 kcal $/ \mathrm{kg}$ body weight, Ensure, Abbott Laboratories) to elicit post-prandial heat production (Panel B). Images were collected at 1-min intervals for the duration of the protocol and representative images are shown in Panel C. Regions of interest (circles) encompass both the supraclavicular BAT depots and the manubrium. Red arrows within the circles indicate the highest temperature in the region of interest, while blue arrows indicate the lowest temperature. 
$3730 \boldsymbol{g}$ for $10 \mathrm{~min}$. To prevent any confounding effect of movement, participants sat upright in a phlebotomy chair for the duration of the resting and imaging periods. The day prior to IR imaging, participants were provided with a standardised meal of beef or vegetarian lasagne (McCain Foods, VIC, Australia) to consume the night before. The two meals were similar in energy and nutritional composition, per serving the beef lasagne provided 1630 kJ energy, $22.8 \mathrm{~g}$ protein, $9.2 \mathrm{~g}$ fat (4.8 g saturated fat), $49.2 \mathrm{~g}$ carbohydrate (16.8 g sugar), whereas the vegetarian option contained $1460 \mathrm{~kJ}$ energy, $19.6 \mathrm{~g}$ protein, $6.0 \mathrm{~g}$ fat (3.6 g saturated) and $50 \mathrm{~g}$ carbohydrate (18.8 g sugar).

\section{Cold- and meal-induced thermogenesis}

The average room temperature $\left(19.36 \pm 0.29^{\circ} \mathrm{C}\right)$ and humidity $(43.35 \pm 1.39 \%)$ were recorded across the experimental period. The FLIR T640 thermal imaging camera (FLIR Systems Australia Pty Ltd, VIC, Australia) was configured $1 \mathrm{~m}$ from the participant and focussed at the level of the clavicles. Emissivity was set to 0.98 as detailed for the measurement of skin temperatures by the FLIR manual. All participants wore a sleeveless shirt that exposed the supraclavicular and manubrium regions, participants changed into this prior to the acclimation period. An outline of the temperature recording protocol is detailed in Fig. 1B. Images were collected at 1-min intervals across the duration of the experiment.

Five baseline images were collected and to induce moderate cold exposure participants immersed one hand into water at $15^{\circ} \mathrm{C}$ for $5 \mathrm{~min}$. Images were collected for a further $30 \mathrm{~min}$ following cold exposure. Once supraclavicular temperature had returned to baseline, another five baseline images were collected, after which participants consumed a standardised liquid meal $(10 \mathrm{kcal} /$ kg body weight, Ensure Powder, Abbott Laboratories). The meal was consumed at room temperature within a 5-min period. Post-prandial temperature recordings were collected for $30 \mathrm{~min}$ (Fig. 1B). Representative thermal images from a single participant from each group are shown in Fig. 1C.

All thermal images were analysed using the program FLIR Tools (FLIR Systems, OR, USA). The maximum temperature within the supraclavicular region was measured bilaterally (Fig. 1C). As a means of negative control within each participant, the minimum temperature of the manubrium, the most superior portion of the sternum, was also measured. The skin of the manubrium overlies the sternum, hence any changes in temperature observed at this site reflects alterations in overall cutaneous heat circulation rather than that caused by thermogenesis such as in BAT and has been used in previous studies as a reference negative control region $(21,22)$. Any image in which the regions of interest were obscured (which occurred during the consumption of the meal replacement drink) were omitted from analysis.

Cold-induced thermogenesis was considered biphasic. Temperature was analysed as the area-under-the-curve (AUC) during cold exposure itself. The mean change in temperature was also calculated at 15-30 min following cold exposure and at 20-30 min post meal consumption.

\section{Blood sample analysis}

A single blood sample was collected from fasted participants to measure plasma concentrations of total cortisol, testosterone, $17 \beta$-estradiol and progesterone.

Plasma cortisol was measured using a ${ }^{3} \mathrm{H}$-based double-antibody RIA as described previously (23), with an intra-assay coefficient of variation (CV) of 3.56\%. Plasma testosterone, $17 \beta$-estradiol and progesterone were all measured using ${ }^{125}$ I-based ImmuChem double antibody RIA kits (MP Biomedicals, OH, USA), with an intra-assay CV of $12.06,8.56$ and $14.02 \%$ respectively.

\section{Statistical analysis}

Statistical analyses were undertaken using IBM SPSS v 22.0. All data were checked for homogeneity and equal variance with Levene's test. Baseline temperature as well as the effects of both cold and meal stimuli on BAT heat production were analysed using a one-way ANOVA and post hoc comparisons were made using the Fisher's least significant difference test. Sex differences were analysed by unpaired Student's $t$-test comparing men to women, irrespective of stage of menstrual cycle. To take into account the effects across time, a repeated-measures two-way ANOVA was performed on the temperature changes observed in the supraclavicular and manubrium regions respectively. The between subject variables were group and sex. Linear regression analyses were used to determine the correlation between plasma cortisol and sex steroid concentrations and all thermogenic measures. Linear regression normality was assessed through the examination of predicted probability (P-P) plots for each relationship and scatterplots of the predicted values versus the residuals for homoscedasticity. All regressions involving testosterone were not normal, so regressions were performed within sex, which restored normality. Statistical significance was taken as a $P<0.05$. Unless otherwise stated, all data are presented as the mean \pm S.E.M. 
Table 1 Anthropometric characteristics of participants.

\begin{tabular}{l}
\hline$n$ \\
Age (years) \\
Height (cm) \\
Weight (kg) \\
BMI (kg/m²) \\
Waist circumference (cm) \\
Adiposity (\%) \\
Fat-free mass (kg) \\
Visceral fat mass (g)
\end{tabular}

\begin{tabular}{c} 
Women - follicular phase \\
\hline 11 \\
$24.64 \pm 1.2$ \\
$164.40 \pm 1.6$ \\
$59.65 \pm 2.2$ \\
$22.12 \pm 0.9$ \\
$71.70 \pm 1.4$ \\
$29.83 \pm 2.5$ \\
$39.66 \pm 1.2$ \\
$104.10 \pm 43.9$ \\
\hline
\end{tabular}

\begin{tabular}{c}
\hline Women - luteal phase \\
\hline 9 \\
$25.22 \pm 1.7$ \\
$162.90 \pm 2.5$ \\
$57.30 \pm 2.2$ \\
$21.56 \pm 0.4$ \\
$68.93 \pm 1.2$ \\
$28.96 \pm 2.5$ \\
$39.00 \pm 1.9$ \\
$49.16 \pm 34.2^{*}$
\end{tabular}

\begin{tabular}{c}
\hline Men \\
\hline 14 \\
$23.07 \pm 0.7$ \\
$180.90 \pm 2.2^{*}$ \\
$76.20 \pm 3.1^{*}$ \\
$23.22 \pm 0.7$ \\
$79.13 \pm 2.0^{*}$ \\
$17.95 \pm 1.6$ \\
$60.06 \pm 2.5^{*}$ \\
$261.30 \pm 74.0$ \\
\hline
\end{tabular}

\begin{tabular}{l}
\multicolumn{1}{c}{$\boldsymbol{P}$ value } \\
\hline NS \\
$<0.0001$ \\
$<0.0001$ \\
$\mathrm{NS}$ \\
$<0.01$ \\
$<0.01$ \\
$<0.0001$ \\
$<0.05$ cf men
\end{tabular}

*indicates a significant difference between one group and the others with the stipulated $P$ value.

\section{Post hoc statistical power calculations}

Priori power calculations were not possible due to the novelty of the longitudinal mode of temperature recording, the method of data analysis, the nature of the stimuli and the inclusion of menstrual cycle as a grouping variable. However, to affirm that a true null hypothesis was not falsely rejected, post hoc statistical power calculations were made using the freely available Statistical Power Calculator Using Average Values from DSS Research (https://www. dssresearch.com/resources/calculators/statistical-powercalculator-average/). All power calculations were performed assuming a two-tailed alpha of 0.05 . Based on study data, the significant difference $\left(0.53^{\circ} \mathrm{C}\right)$ between women measured during the luteal phase and those during the follicular phase, was $100 \%$ powered. The difference between men and women with regards to post-cold adaptive and mealinduced thermogenesis had a power of 95.9 and $97.9 \%$. Thus the group numbers attained were adequate to detect the fundamental differences in thermogenesis between men and women.

\section{Results}

\section{Participant characteristics}

Thirty-four young, healthy participants attended and completed all components of the study. Women during the follicular phase $(n=11)$ or the luteal phase $(n=9)$ of the menstrual cycle and 14 men were studied. The average age of participants was $24.1 \pm 0.65$ years, with no difference between men $(23.1 \pm 0.7$ years $)$ and women (follicular phase: $24.6 \pm 1.2$ years, luteal phase: $25.22 \pm 1.7$ years). Morphometric characteristics are described in Table 1. Plasma $17 \beta$-estradiol concentrations were lower $(P<0.01)$ and testosterone concentrations were higher $(P<0.0001)$ in men than in women, irrespective of the stage of menstrual cycle (Table 2). Concentrations of $17 \beta$-estradiol were similar in women during the luteal and follicular phases of the menstrual cycle and progesterone concentrations were higher $(P<0.0001)$ during the luteal phase, than in women during follicular phase and in men (Table 2). Basal plasma cortisol concentrations were similar in men and women, but were lower $(P<0.05)$ in women during luteal phase than during the follicular phase of the menstrual cycle (Table 2).

\section{Basal BAT temperature was elevated in women during the luteal phase of the menstrual cycle}

Baseline supraclavicular temperature was greater $(P<0.05)$ in women during the luteal phase than in women during the follicular phase of the menstrual cycle and in men (Fig. 2A). This difference was specific to the BAT region as the manubrium temperature was similar across all 3 groups (Fig. 2B). Baseline supraclavicular temperature was inversely

Table 2 Plasma concentration of steroids.

\begin{tabular}{lc}
\hline Group & Follicular phase women \\
\cline { 1 - 2 } $17 \beta$-estradiol $(\mathrm{pg} / \mathrm{mL})$ & 11 \\
Progesterone $(\mathrm{ng} / \mathrm{mL})$ & $145.0 \pm 34.2$ \\
Testosterone $(\mathrm{ng} / \mathrm{mL})$ & $0.3 \pm 0.1$ \\
Cortisol $(\mathrm{ng} / \mathrm{mL})$ & $0.3 \pm 0.0$ \\
\hline
\end{tabular}

\begin{tabular}{c}
\hline Luteal phase women \\
\hline 9 \\
$195.9 \pm 34.6$ \\
$11.2 \pm 2.8^{*}$ \\
$0.3 \pm 0.0$ \\
$215.9 \pm 18.9$
\end{tabular}

\begin{tabular}{c}
\hline Men \\
\hline 14 \\
$30.1 \pm 3.7^{*}$ \\
$0.2 \pm 0.0$ \\
$4.4 \pm 0.3^{*}$ \\
$273.5 \pm 38.3$
\end{tabular}

P value
0.01
0.0001
0.0001
0.05 cf luteal

*indicates a significant difference between one group and the others with the stipulated $P$ value. 

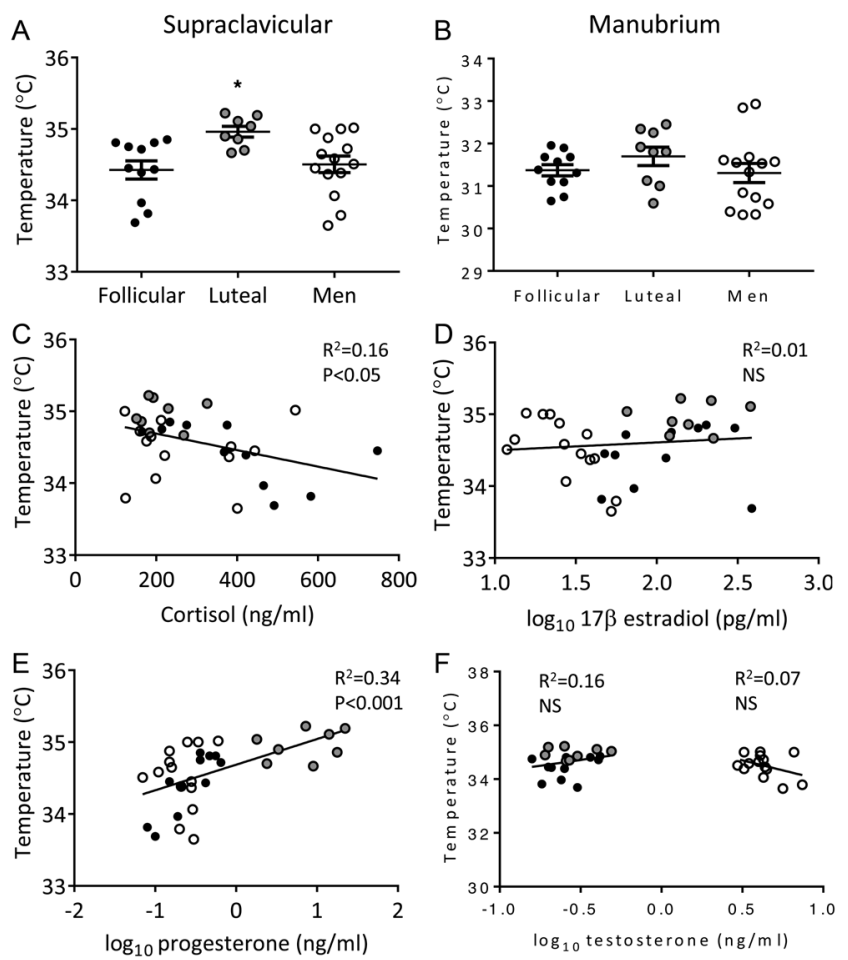

- Follicular phase women

- Luteal phase women

o Men

\section{Figure 2}

Baseline supraclavicular temperature was higher in women during the luteal phase, which correlated with progesterone as well as cortisol. The mean temperature of supraclavicular BAT across pre-cold and pre-meal baseline measurement periods was greatest in women during the luteal phase of the menstrual cycle $(A)$, yet no difference in temperature was observed at the manubrium (B). Through simple linear regression, the relationship between baseline BAT temperatures and cortisol (B), 17 $\beta$-estradiol (D), progesterone (E) and testosterone (F). Indication of grouping among regression analysis is only for qualitative purposes. A logarithmic transformation was applied to the variables $17 \beta$-estradiol, testosterone and progesterone. The squared correlation coefficient $\left(R^{2}\right)$ and $P$ value $(P)$ are indicated except in cases of the $P$ value exceeding 0.1 , in which case only non-significance (NS) is indicated. Data presented as the mean \pm S.E.M. * indicates a significant difference of $P<0.05$ as determined via a one-way ANOVA with post hoc Fisher's least significant difference test.

correlated $\left(\beta=-0.001, \quad R^{2}=0.16\right)$ with plasma cortisol concentration (Fig. 2C) and positively correlated $(\beta=0.36$, $R^{2}=0.34$ ) with plasma progesterone concentration (Fig. $2 \mathrm{E}$ ). Baseline supraclavicular temperature did not correlate with either $17 \beta$-estradiol or testosterone concentrations
(Fig. 2D and F). There was no association between baseline manubrium temperature and the concentration of any of the sex steroids or cortisol (Table 3).

\section{Cold-induced thermogenesis is abrogated in women during the follicular phase of the menstrual cycle}

Temperature responses to cold exposure in the supraclavicular and manubrium regions are shown in Fig. 3. None of the participants reported shivering during or after the moderate cold stimulus. There was no effect of mild acute cold exposure on manubrium temperature (Fig. 3A), whereas the supraclavicular temperature increased (Fig. 3B), indicating a specific effect of cold-stimulus on BAT. During the cold stimulus itself, supraclavicular temperature increased in all three groups, albeit to varying degrees. The AUC was greater $(P<0.05)$ in men and in women during luteal phase than in women during the follicular phase of the menstrual cycle $(1.66 \pm 0.44$ AUC in men, $1.56 \pm 0.26$ AUC in women during luteal phase, $0.50 \pm 0.23$ AUC in women during the follicular phase; Fig. 3B and C). The AUC of the temperature change in the manubrium did not differ between groups (Fig. 3E).

\section{Cold-induced adaptive thermogenesis is greater in women than men}

After cold exposure, supraclavicular temperature initially returned to baseline and then increased again approximately 20 min after the cessation of the cold exposure period (Fig. 3B); this response will be referred to as the cold-induced adaptive response. The adaptive increase in supraclavicular temperature was evident in women, but appeared to be absent in men $\left(P<0.05 ; 0.16 \pm 0.04^{\circ} \mathrm{C}\right.$ increase in women vs $0.00 \pm 0.08^{\circ} \mathrm{C}$ in men), irrespective of the stage in the menstrual cycle (Fig. 3B and D). This was not observed in the manubrium (Fig. 3A and F).

\section{Sex steroids are associated with cold-induced adaptive thermogenesis}

Despite the apparent effect of the stage of menstrual cycle on BAT temperature during cold exposure, there was no significant association between the cold-induced AUC response and plasma concentrations of cortisol, progesterone, $17 \beta$-estradiol or testosterone (Fig. 3G, H, I and $\mathrm{J})$. In contrast, cold-induced adaptive thermogenesis was positively correlated $\left(\beta=0.20, R^{2}=0.13, P<0.05\right)$ with plasma 
Table 3 Association between manubrium temperature responses and plasma steroid concentrations.

\begin{tabular}{|c|c|c|c|c|c|c|c|c|}
\hline \multirow[b]{2}{*}{ Independent variables } & \multicolumn{2}{|c|}{ Baseline temperature } & \multicolumn{2}{|c|}{ Cold response AUC } & \multicolumn{2}{|c|}{$\begin{array}{l}\text { Cold adaptive } \\
\text { response mean }\end{array}$} & \multicolumn{2}{|c|}{$\begin{array}{c}\text { Post-meal } \\
\text { response AUC }\end{array}$} \\
\hline & $R^{2}$ & $P$ & $R^{2}$ & $P$ & $R^{2}$ & $P$ & $R^{2}$ & $P$ \\
\hline Cortisol & 0.03 & 0.34 & 0.01 & 0.65 & 0.14 & 0.03 & 0.01 & 0.53 \\
\hline Progesterone & 0.02 & 0.42 & 0.00 & 0.82 & 0.00 & 0.80 & 0.01 & 0.64 \\
\hline $17 \beta$-estradiol & 0.01 & 0.60 & 0.09 & 0.09 & 0.02 & 0.44 & 0.02 & 0.46 \\
\hline \multicolumn{9}{|l|}{ Testosterone } \\
\hline Men & 0.07 & 0.36 & 0.34 & 0.03 & 0.13 & 0.20 & 0.00 & 0.85 \\
\hline Women & 0.06 & 0.29 & 0.01 & 0.70 & 0.00 & 0.78 & 0.00 & 0.79 \\
\hline
\end{tabular}

*Regressions with $P<0.05$ are highlighted in bold.

17ß-estradiol levels (Fig. 3L). There was no association between the cold-induced adaptive change in supraclavicular temperature and plasma concentrations of either cortisol, progesterone or testosterone (Fig. $3 \mathrm{~K}, \mathrm{M}$ and $\mathrm{N}$ ).

\section{Meal-induced thermogenesis is higher in women than men}

Changes in the temperature of the supraclavicular region and the manubrium in response to a standardised liquid meal are displayed in Fig. 4. The temperature of both the supraclavicular and the manubrium increased approximately $15 \mathrm{~min}$ after meal consumption (Fig. 4A and $\mathrm{B})$. The meal-induced increase in supraclavicular temperature was greater in women than men, irrespective of the stage of the menstrual cycle. The difference was determined by measuring both the change in AUC $(2.29 \pm 0.33$ AUC in women vs $0.97 \pm 0.52$ AUC in men; Fig. 4C) and the post-prandial change in supraclavicular temperature $\left(0.23 \pm 0.03^{\circ} \mathrm{C}\right.$ in women vs $0.11 \pm 0.06^{\circ} \mathrm{C}$ in men; Fig. 4D). In contrast, the manubrium temperature was similar in men and women (Figs. 4A, E and F).

\section{$17 \beta$-Estradiol and testosterone levels predict meal-induced thermogenesis}

There was a weak correlation between the supraclavicular temperature response to meal ingestion (AUC) and plasma $17 \beta$-estradiol concentration $\left(\beta=1.28, R^{2}=0.10, P=0.07\right)$ (Fig. $4 \mathrm{H})$, whereas there was no association with the change in supraclavicular temperature and plasma cortisol, progesterone or testosterone levels (Fig. 4G, I and J).

\section{Discussion}

The regulation of body temperature is sexually dimorphic and is influenced by fluctuating sex steroid concentrations
(17). Furthermore, retrospective studies indicate that women may possess more functional BAT than men (12, $24,25)$, but no studies have heretofore determined whether BAT activity changes across the menstrual cycle in women. The present study employed infrared thermography to demonstrate that BAT heat production in response to both cold exposure and meal consumption was greater in women than in men. Furthermore, we demonstrate that cold-induced thermogenesis varies across the menstrual cycle, such that activation of BAT is attenuated during the follicular phase relative to the luteal phase of the cycle. Thus, sex and the stage of menstrual cycle impact on BAT activity in healthy women of normal body weight.

It is well known that body temperature fluctuates in women across the menstrual cycle and is higher during the luteal phase than either the follicular or ovulatory phases $(17,26)$. A recent study utilised wrist temperature biosensors to demonstrate that night time cutaneous temperature was elevated by $0.33^{\circ} \mathrm{C}$ during the early luteal phase compared to the period of ovulation (27). The present study further demonstrates that, in fasted subjects, baseline BAT temperature is greater in women during the luteal phase than in women during the follicular phase and in men. Sex differences with respect to thermoregulation have been acknowledged for over 50 years, whereby women maintain a greater core body temperature during cold exposure (28, 29). Despite this, women experience lower cutaneous temperature during cold exposure, which coincides with increased perception of cold compared to men (28). As indicated above, retrospective analysis of PET-CT scans have shown active BAT is more readily detected in women than in men (12), but these studies did not relate such findings to fluctuations in sex steroids or stage of menstrual cycle. To our knowledge, the present study is the first to describe elevated basal BAT temperatures in women during the luteal phase.

In addition to the differences in baseline BAT temperature, we demonstrate that both sex and the stage 


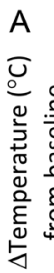

Manubrium

- Follicular Phase Women

-o Luteal Phase Women

-o- Men
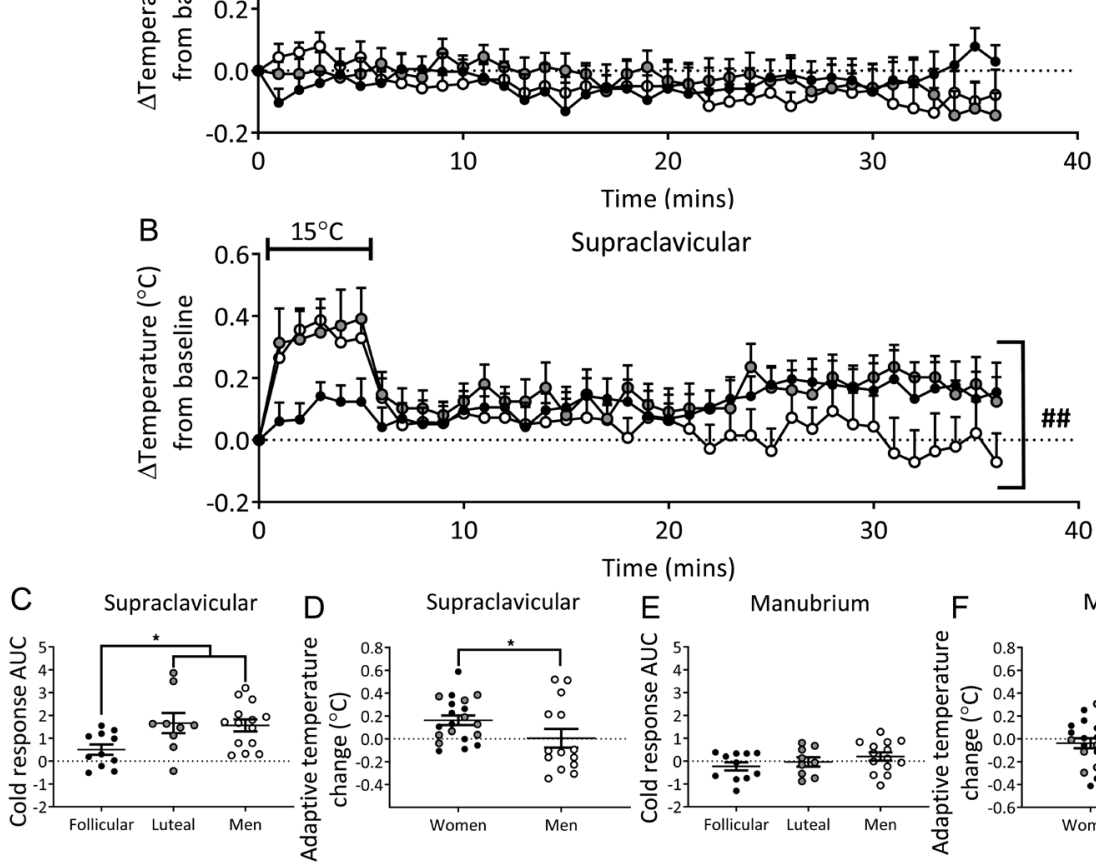

Time (mins)
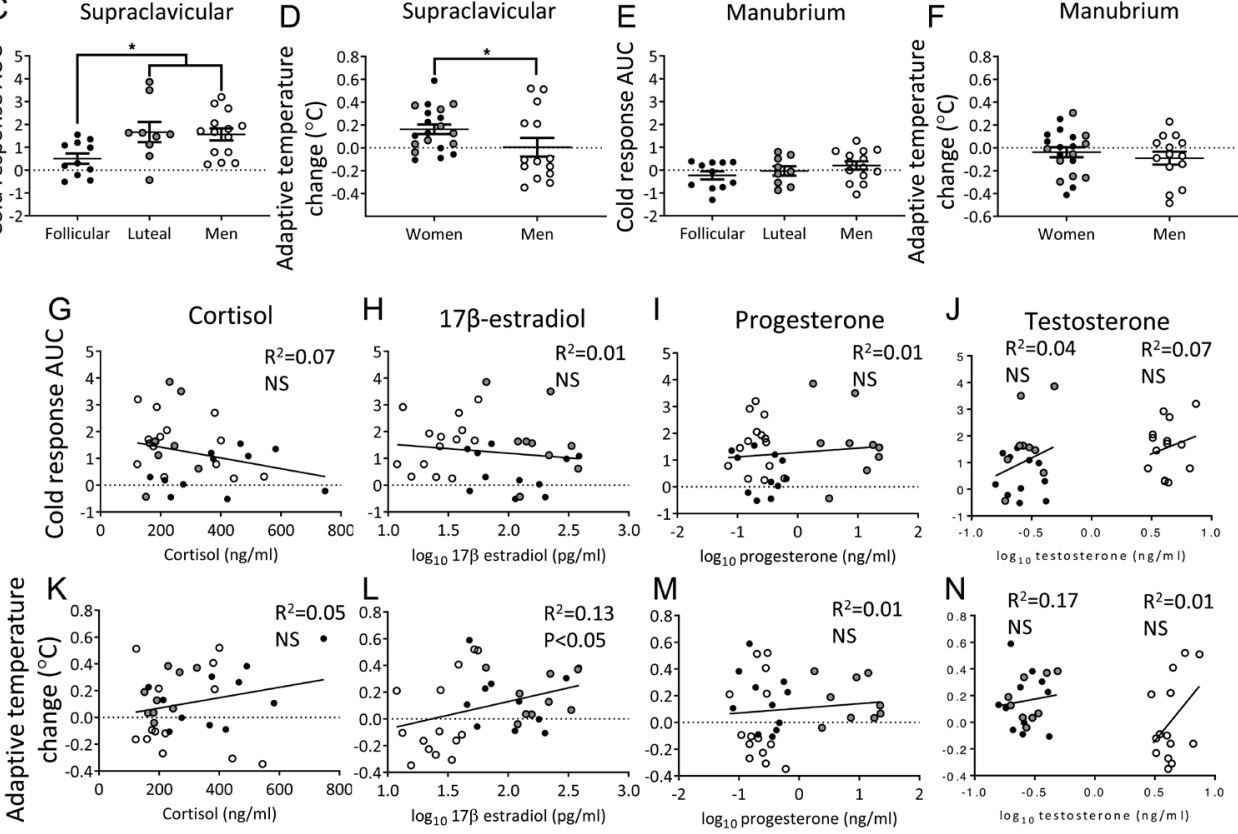

\section{Figure 3}

Cold-induced thermogenesis in men $(n=14)$ and women during the luteal phase of the menstrual cycle $(n=9)$ was greater than in women during the follicular phase $(n=11)$. Temperature changes during cold exposure (hand immersed in water at $15^{\circ} \mathrm{C}$ ) and for 30 min thereafter were tracked at the manubrium (A) and BAT (B). The area under the curve (AUC) was calculated over the course of the cold exposure temperature response (0-5 min) in BAT (C) and the manubrium (E). The mean temperature changes were calculated at 20-35 min after cold exposure, which was in line with the adaptive thermogenic response in men and women at both BAT (D) and manubrium (F). Simple linear regressions were established between measured steroid hormones and cold response AUC (G-J) as well as the cold-induced adaptive temperature change (K, L, M and N). In the case of the regressions, indication of grouping is purely for qualitative purposes. A logarithmic transformation was applied to the variables $17 \beta$-estradiol, testosterone and progesterone. The squared correlation coefficient $\left(R^{2}\right)$ and $P$ value $(P)$ are indicated except in cases of the $P$ value exceeding 0.1, in which case only non-significance (NS) is indicated. Data presented as the mean \pm S.E.M. * indicates a significant difference of $P<0.05$ as determined with an unpaired Student's $t$-test or a one-way ANOVA with post hoc Fisher's least significant difference test where appropriate. \#\# indicates an effect of sex with $P<0.01$ as determined with a repeated measures two-way ANOVA with post hoc Fisher's least significant difference test. 
A Meal (10kcal $/ \mathrm{kg}$ body weight)
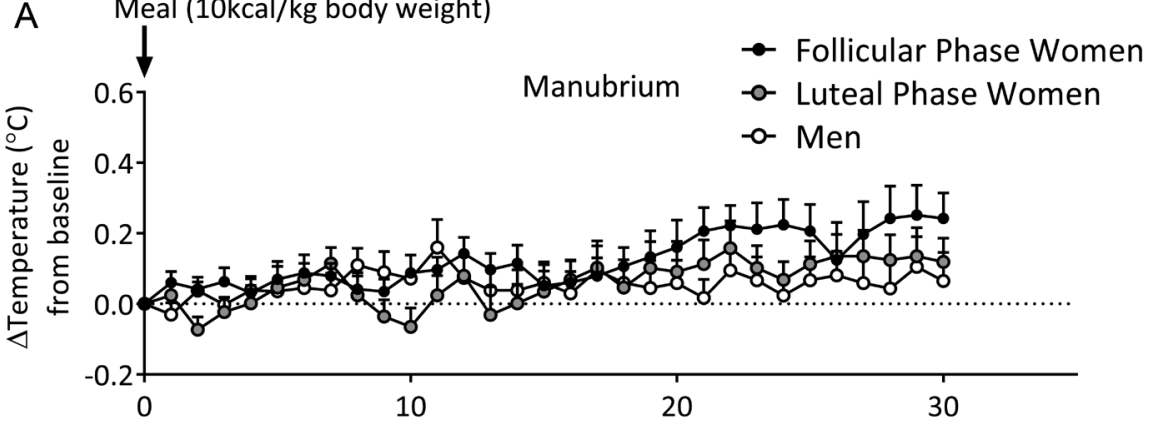

B Meal (10kcal/kg body weight)

Time (mins)
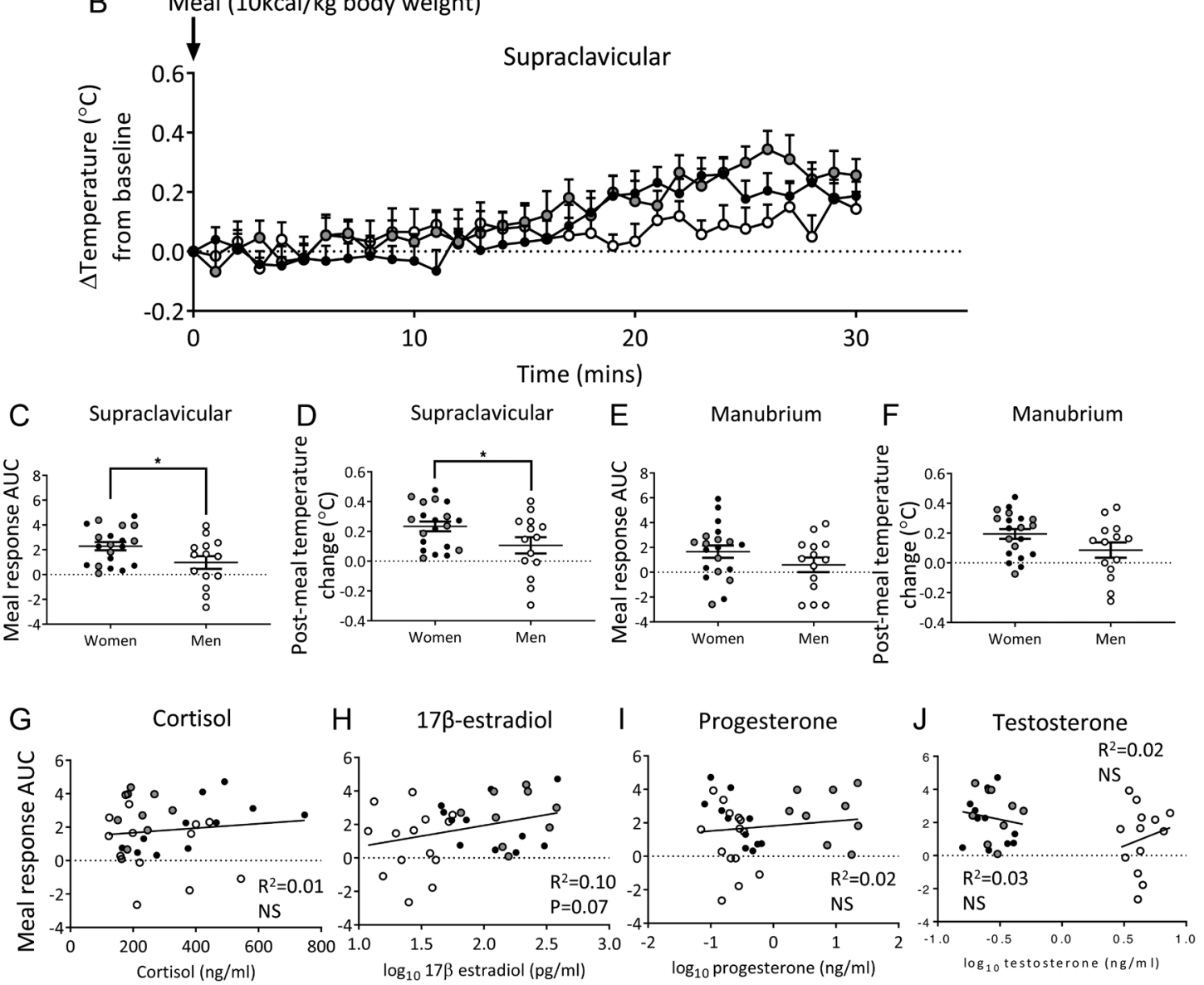

\section{Figure 4}

Meal-induced thermogenesis was higher in women during either follicular $(n=11)$ or luteal $(n=9)$ phases of the menstrual cycle than men $(n=14)$. Temperature changes were tracked from baseline for 30 min following meal-replacement drink administration at the manubrium (A) and BAT (B). The area under the curve (AUC) was calculated for the temperature changes during the final 10 min of post-meal thermogenesis (20-30 min) in both men and women for both the supraclavicular (C) and manubrium (E). The mean temperature changes at the supraclavicular (D) and manubrium (F), during the final 10 min of post-meal thermogenesis (20-30 $\mathrm{min}$ ) are presented in both men and women. Simple linear regressions were established between measured steroid hormones and meal response $A \cup C(G, H, I$ and $\mathrm{J})$. The indication of grouping among regressions is for qualitative purposes. $A$ logarithmic transformation was applied to the variables $17 \beta$-estradiol, testosterone and progesterone. The squared correlation coefficient $\left(R^{2}\right)$ and $P$ value $(P)$ are indicated except in cases of the $P$ value exceeding 0.1 , in which case only non-significance (NS) is indicated. Data presented as the mean \pm S.E.M. * indicates a significant difference of $P<0.05$ as determined with an unpaired Student's t-test. 
of menstrual cycle impact on the activation of BAT in response to either a cold stimulus or a meal stimulus. Thermogenic responses to cold stimuli were biphasic and were segregated into two responses, being firstly, the response during the period of cold stimulus and the adaptive response occurring approximately $20 \mathrm{~min}$ after cessation of the stimulus. Importantly, temperature changes in response to cold exposure were specific to the supraclavicular region, with no effect on the temperature of the manubrium, suggesting that responses were specific to BAT and were not caused by a generalised increase in core body temperature. During cold exposure, BAT temperature increased in both men and women, but this effect was attenuated in women during the follicular phase of the menstrual cycle. Previous studies have shown that, in response to very mild cold exposure $\left(23^{\circ} \mathrm{C}\right)$, the increase in metabolic rate is also attenuated in women during the follicular phase relative to the luteal phase of the menstrual cycle (30). Indeed, a recent cold acclimation study suggested that ${ }^{18} \mathrm{~F}$-FDG uptake within the supraclavicular region was similar in men and women, but this work only characterised women during the follicular phase of the menstrual cycle (15). Together these studies, along with the current work, highlight the importance of the stage of menstrual cycle and thus the possible role of endogenous sex steroids in modulating metabolic responses to cold. Despite this, the vast majority of studies characterising BAT function in humans are predominantly in men and do not account for the stage of menstrual cycle in women on the occasions when they are included $(31,32)$.

Supraclavicular temperature increased approximately 20 min after either meal intake or cold stimulus, indicative of adaptive thermogenesis. In contrast to cold exposure when the temperature increase was localised to the supraclavicular region, a generalised increase in temperature was observed in both the supraclavicular and manubrium regions following meal consumption. Various studies in rodents, show BAT thermogenesis to be increased in response to intake of a single meal (33, $34)$, but the role of BAT in diet-induced thermogenesis in humans has been contentious. In contrast, work using PET-CT demonstrated that BAT thermogenesis was increased after consumption of a carbohydrate enriched mixed meal (35). Furthermore, the increase in BAT activity was correlated with increased energy expenditure during the post-prandial period (35). Herein, we demonstrate that consumption of a standardised mixed liquid (Ensure) meal increases supraclavicular temperature in men and women, indicative of post-prandial thermogenesis in BAT. Because this was also associated with an increase in temperature of the manubrium, the response is generalised and cannot be regarded as specifically related to BAT thermogenesis and in fact may relate to altered skin perfusion. Nonetheless, our previous work in sheep demonstrated that skeletal muscle mounts a post-prandial temperature response without an associated change in blood flow, which was taken to indicate thermogenesis in this tissue (36). Further studies are required to determine the differential contribution of skeletal muscle and BAT (and/or other tissues) to the post-prandial rise in temperature in humans.

It is important to emphasise that the adaptive thermogenic responses to both meal and cold stimuli differed in men and women, irrespective of the stage of the menstrual cycle; the increase in supraclavicular temperature was greater in women than men. This is consistent with the observation that there is greater BAT volume and activity in women than in men under thermoneutral conditions (25). To further elucidate the effect of sex and stage of menstrual cycle on BAT temperature and activation we sought to characterise the possible relationship between supraclavicular temperature and plasma concentrations of cortisol, progesterone, $17 \beta$-estradiol and testosterone. We demonstrate that baseline supraclavicular temperature was positively correlated with plasma progesterone levels, but there was a negative association with plasma cortisol concentration. Previous studies in human brown adipocytes show that dexamethasone treatment blocks adrenergic stimulation of UCP1 expression (37). Furthermore, prednisolone treatment attenuates both cold-induced and post-prandial thermogenesis (38). In the current study, there was no association with plasma cortisol concentration and innate variation in either diet- or cold-induced supraclavicular heat production. Despite this, we demonstrate that circulating cortisol is associated with variations in basal supraclavicular temperature, whereby endogenous cortisol may negatively regulate basal BAT activity.

Progesterone is considered to be the primary driver of the thermoregulatory vicissitudes across the menstrual cycle (39), perhaps by reducing cutaneous circulation with a consequent increase in core body temperature $(40,41)$ or via thermosensitive neurons within the preoptic area of the hypothalamus as observed in rats (42). Previous studies have suggested that during the luteal phase, women defend a higher body temperature and therefore exhibit increased shivering and greater vasodilation in response to cold exposure (18). In addition, in response to lower ambient temperature, women exhibit an exacerbated increase in energy expenditure during the luteal phase compared 
to those during the follicular phase, despite showing no differences in perception of cold (43). As indicated earlier, we demonstrate supraclavicular temperature was greater in women during the luteal phase than in women during the follicular phase and that this correlated with plasma progesterone levels. Therefore, an increase in basal BAT activity may also contribute to increased body temperature in the luteal phase (39), and furthermore, greater activation of BAT may be a physiological means to maintain the increased core body temperature during episodes of cold exposure.

The adaptive supraclavicular temperature responses to both cold- and meal-stimuli were positively correlated with plasma $17 \beta$-estradiol levels. Although there is a paucity of studies in humans, sex steroids have been shown to modulate thermogenesis in various animal models, such that estrogens are known to increase BAT activity (13, 44 ). In rodents, $17 \beta$-estradiol is thought to inhibit AMPK neurons within the ventromedial hypothalamus, leading to sympathetic activation of BAT (45). This supports the hypothesis that inherent differences in BAT thermogenesis between healthy men and women are due, at least in part, to variation in the peripheral concentration of estrogen. Despite the association between supraclavicular temperature and female sex steroids, there was no association with circulating testosterone concentrations and any indices of BAT thermogenesis. This is consistent with our previous work demonstrating that clinically elevated testosterone levels are correlated with reduced supraclavicular temperature in women with polycystic ovarian syndrome, but there is no association between the two measurements in control women (46); further work is required to elucidate any possible effects of androgens on BAT function.

In conclusion, we demonstrate sexual dimorphism in basal supraclavicular temperature and in response to both cold and dietary stimuli. However, to our knowledge, this is the first study that has sought to link these differences with endogenous circulating concentrations of sex steroids in humans. Herein we have shown that the female sex steroids, progesterone and $17 \beta$-estradiol are associated with innate differences in basal supraclavicular temperature and the temperature responses to thermogenic stimuli, respectively. Furthermore we demonstrate that the stage of menstrual cycle can impact on temperature responses during cold exposure, an effect that appears to be independent of sex steroids. As eluded to above the vast majority of previous studies investigating BAT physiology are carried out in men, and thus this highlights the fundamental importance to investigate sex differences as well as the impact of the stage of menstrual cycle on BAT activation and energy expenditure.

\section{Declaration of interest}

The authors declare that there is no conflict of interest that could be perceived as prejudicing the impartiality of this study.

\section{Funding}

This work was funded by a Monash University Advancing Women's Success in Science grant awarded to Dr Belinda Henry

\section{Author contribution statement}

The study was designed by B A Henry, J P Fuller-Jackson and I J Clarke. Data analyses was performed by J P Fuller-Jackson and B A Henry. All authors contributed to the preparation of the manuscript.

\section{References}

1 van Marken Lichtenbelt WD \& Schrauwen P. Implications of nonshivering thermogenesis for energy balance regulation in humans. American Journal of Physiology: Regulatory, Integrative and Comparative Physiology. 2011301 R285-R296. (https://doi. org/10.1152/ajpregu.00652.2010)

2 Betz MJ \& Enerbäck S. Targeting thermogenesis in brown fat and muscle to treat obesity and metabolic disease. Nature Reviews Endocrinology 2017 14 77. (https://doi.org/10.1038/nrendo.2017.132)

3 van Marken Lichtenbelt WD, Vanhommerig JW, Smulders NM, Drossaerts JMAFL, Kemerink GJ, Bouvy ND, Schrauwen P \& Jaap Teule GJ. Cold activated brown adipose tissue in healthy men. New England Journal of Medicine 2009360 1500-1508. (https://doi. org/10.1056/NEJMoa0808718)

4 Cypess AM, White AP, Vernochet C, Schulz TJ, Xue R, Sass CA, Huang TL, Roberts-Toler C, Weiner LS \& Sze C. Anatomical localization, gene expression profiling and functional characterization of adult human neck brown fat. Nature Medicine 201319 635-639. (https://doi.org/10.1038/nm.3112)

$5 \mathrm{Wu}$ J, Bostrom P, Sparks LM, Ye L, Choi JH, Giang AH, Khandekar M, Virtanen KA, Nuutila P, Schaart G et al. Beige adipocytes are a distinct type of thermogenic fat cell in mouse and human. Cell $2012 \mathbf{1 5 0}$ 366-376. (https://doi.org/10.1016/j.cell.2012.05.016)

6 Schulz TJ, Huang TL, Tran TT, Zhang H, Townsend KL, Shadrach JL, Cerletti M, McDougall LE, Giorgadze N, Tchkonia T et al. Identification of inducible brown adipocyte progenitors residing in skeletal muscle and white fat. PNAS 2011108 143-148. (https://doi. org/10.1073/pnas.1010929108)

7 Barbatelli G, Murano I, Madsen L, Hao Q, Jimenez M, Kristiansen K, Giacobino JP, De Matteis R \& Cinti S. The emergence of coldinduced brown adipocytes in mouse white fat depots is determined predominantly by white to brown adipocyte transdifferentiation. American Journal of Physiology: Endocrinology and Metabolism 2010298 E1244-E1253. (https://doi.org/10.1152/ajpendo.00600.2009)

8 Yoneshiro T, Aita S, Matsushita M, Kayahara T, Kameya T, Kawai Y, Iwanaga $\mathrm{T} \&$ Saito M. Recruited brown adipose tissue as an antiobesity agent in humans. Journal of Clinical Investigation 2013 123 3404-3408. (https://doi.org/10.1172/JCI67803)

9 Hanssen MJ, Hoeks J, Brans B, van der Lans AA, Schaart G, van den Driessche JJ, Jorgensen JA, Boekschoten MV, Hesselink MK, Havekes B et al. Short-term cold acclimation improves insulin sensitivity in patients with type 2 diabetes mellitus. Nature Medicine 201521 863-865. (https://doi.org/10.1038/nm.3891) 
10 Bartelt A, Bruegelmann K, Bruns OT, Eychmuller A, Freund B, Gordts PLSM, Heeren J, Hohenberg H, Ittrich H, Kaul MG et al. Brown adipose tissue activity controls triglyceride clearance. Nature Medicine 201117 200-205. (https://doi.org/10.1038/nm.2297)

11 Oliver P, Lombardi A \& De Matteis R. Editorial: insights into brown adipose tissue functions and browning phenomenon. Frontiers in Physiology 2020 11. (https://doi.org/10.3389/fphys.2020.00219)

12 Ouellet V, Routhier-Labadie A, Bellemare W, Lakhal-Chaieb L, Turcotte E, Carpentier AC \& Richard D. Outdoor temperature, age, sex, body mass index, and diabetic status determine the prevalence, mass, and glucose-uptake activity of 18F-FDG-detected BAT in humans. Journal of Clinical Endocrinology and Metabolism 201196 192-199. (https://doi.org/10.1210/jc.2010-0989)

13 Martinez de Morentin PB, Gonzalez-Garcia I, Martins L, Lage R, Fernandez-Mallo D, Martinez-Sanchez N, Ruiz-Pino F, Liu J, Morgan DA, Pinilla L et al. Estradiol regulates brown adipose tissue thermogenesis via hypothalamic AMPK. Cell Metabolism 201420 41-53. (https://doi.org/10.1038/nrendo.2017.132)(https://doi. org/10.1016/j.cmet.2014.03.031)

14 Clarke SD, Clarke IJ, Rao A, Evans RG \& Henry BA. Differential effects of acute and chronic estrogen treatment on thermogenic and metabolic pathways in ovariectomized sheep. Endocrinology 2013154 184-192. (https://doi.org/10.1210/en.2012-1758)

15 Fletcher LA, Kim K, Leitner BP, Cassimatis TM, O'Mara AE, Johnson JW, Halprin MS, McGehee SM, Brychta RJ, Cypess AM et al. Sexual dimorphisms in adult human brown adipose tissue. Obesity 202028 241-246. (https://doi.org/10.1002/oby.22698)

16 Olsen JM, Csikasz RI, Dehvari N, Lu L, Sandstrom A, Oberg AI, Nedergaard J, Stone-Elander S \& Bengtsson T. beta3-adrenergically induced glucose uptake in brown adipose tissue is independent of UCP1 presence or activity: mediation through the mTOR pathway. Molecular Metabolism 20176 611-619. (https://doi.org/10.1016/j. molmet.2017.02.006)

17 Marshall J. Thermal changes in the normal menstrual cycle. BMJ 19631 102-104. (https://doi.org/10.1136/bmj.1.5323.102)

18 Hessemer V \& Bruck K. Influence of menstrual cycle on shivering, skin blood flow, and sweating responses measured at night. Journal of Applied Physiology 198559 1902-1910.

19 Rodríguez AM, Monjo M, Roca P \& Palou A. Opposite actions of testosterone and progesterone on UCP1 mRNA expression in cultured brown adipocytes. Cellular and Molecular Life Sciences 2002 59 1714-1723. (https://doi.org/10.1152/jappl.1985.59.6.1902)

20 Monjo M, Rodríguez AM, Palou A \& Roca P. Direct effects of testosterone, $17 \beta$-estradiol, and progesterone on adrenergic regulation in cultured brown adipocytes: potential mechanism for gender-dependent thermogenesis. Endocrinology 2003144 4923-4930. (https://doi.org/10.1210/en.2003-0537)

21 El Hadi H, Frascati A, Granzotto M, Silvestrin V, Ferlini E, Vettor R $\&$ Rossato M. Infrared thermography for indirect assessment of activation of brown adipose tissue in lean and obese male subjects. Physiological Measurement 201637 N118-N128. (https://doi. org/10.1088/0967-3334/37/12/N118)

22 Thuzar M, Law WP, Ratnasingam J, Jang C, Dimeski G \& Ho KKY Glucocorticoids suppress brown adipose tissue function in humans: a double-blind placebo-controlled study. Diabetes, Obesity and Metabolism 201820 840-848. (https://doi.org/10.1111/dom.13157)

23 Bocking AD, McMillen IC, Harding R \& Thorburn GD. Effect of reduced uterine blood flow on fetal and maternal cortisol. Journal of Developmental Physiology 19868 237-245. (https://doi.org/10.1152/ ajpendo.00298.201)

24 Lee P, Greenfield JR, Ho KK \& Fulham MJ. A critical appraisal of the prevalence and metabolic significance of brown adipose tissue in adult humans. American Journal of Physiology, Endocrinology and Metabolism 2010299 E601-E606.

25 Pfannenberg C, Werner MK, Ripkens S, Stef I, Deckert A, Schmadl M, Reimold M, Haring HU, Claussen CD \& Stefan N. Impact of age on the relationships of brown adipose tissue with sex and adiposity in humans. Diabetes 201059 1789-1793. (https://doi.org/10.2337/ db10-0004)

26 Baker FC, Waner JI, Vieira EF, Taylor SR, Driver HS \& Mitchell D. Sleep and 24 hour body temperatures: a comparison in young men, naturally cycling women and women taking hormonal contraceptives. Journal of Physiology 2001530 565-574. (https://doi. org/10.1111/j.1469-7793.2001.0565k.x)

27 Shilaih M, Goodale Brianna M, Falco L, Kübler F, De Clerck V \& Leeners B. Modern fertility awareness methods: wrist wearables capture the changes in temperature associated with the menstrual cycle. Bioscience Reports 201838 BSR20171279. (https://doi. org/10.1042/BSR20171279)

28 Cunningham DJ, Stolwijk JA \& Wenger CB. Comparative thermoregulatory responses of resting men and women. Journal of Applied Physiology: Respiratory, Environmental and Exercise Physiology 197845 908-915. (https://doi.org/10.1152/jappl.1978.45.6.908)

29 Hardy JD \& Du Bois EF. Differences between men and women in their response to heat and cold. PNAS 194026 389-398.

30 Nagashima K. Thermoregulation and menstrual cycle. Temperature 20152 320-321. (https://doi.org/10.1080/23328940.2015.1066926)

31 Bloor ID \& Symonds ME. Sexual dimorphism in white and brown adipose tissue with obesity and inflammation. Hormones and Behavior 201466 95-103. (https://doi.org/10.1016/j. yhbeh.2014.02.007)

32 Law J, Bloor I, Budge H \& Symonds ME. The influence of sex steroids on adipose tissue growth and function. Hormone Molecular Biology and Clinical Investigation 201419 13-24. (https://doi.org/10.1515/ hmbci-2014-0015)

33 Allard M \& Leblanc J. Effects of cold acclimation, cold exposure, and palatability on postprandial thermogenesis in rats. International Journal of Obesity 198812 169-178.

34 Glick Z. Inverse relationship between brown fat thermogenesis and meal size: the thermostatic control of food intake revisited. Physiology and Behavior 198229 1137-1140. (https://doi.org/10.1016/00319384(82)90310-9)

35 U Din M, Saari T, Raiko J, Kudomi N, Maurer SF, Lahesmaa M, Fromme T, Amri EZ, Klingenspor M, Solin O et al. Postprandial oxidative metabolism of human brown fat indicates thermogenesis. Cell Metabolism 201828 207.e3-216.e3. (https://doi.org/10.1016/j. cmet.2018.05.020)

36 Clarke SD, Lee K, Andrews ZB, Bischof R, Fahri F, Evans RG, Clarke IJ $\&$ Henry BA. Postprandial heat production in skeletal muscle is associated with altered mitochondrial function and altered futile calcium cycling. American Journal of Physiology: Regulatory, Integrative and Comparative Physiology 2012303 R1071-R1079. (https://doi. org/10.1152/ajpregu.00036.2012)

37 Barclay JL, Agada H, Jang C, Ward M, Wetzig N \& Ho KK. Effects of glucocorticoids on human brown adipocytes. Journal of Endocrinology 2015224 139-147. (https://doi.org/10.1530/JOE-14-0538)

38 Thuzar M, Law WP, Ratnasingam J, Jang C, Dimeski G \& Ho KKY. Glucocorticoids suppress brown adipose tissue function in humans: a double-blind placebo-controlled study. Diabetes, Obesity and Metabolism 201820 840-848. (https://doi.org/10.1111/dom.13157)

39 Cagnacci A, Volpe A, Paoletti AM \& Melis GB. Regulation of the 24-hour rhythm of body temperature in menstrual cycles with spontaneous and gonadotropin-induced ovulation. Fertility and Sterility 199768 421-425. (https://doi.org/10.1016/s00150282(97)00242-2)

40 Charkoudian N \& Johnson JM. Reflex control of cutaneous vasoconstrictor system is reset by exogenous female reproductive hormones. Journal of Applied Physiology (1985) 199987 381-385. (https://doi.org/10.1152/jappl.1999.87.1.381)

41 Charkoudian N \& Stachenfeld NS. Reproductive hormone influences on thermoregulation in women. Comprehensive Physiology 20144 793-804. (https://doi.org/10.1002/cphy.c130029) 
42 Tsai CL, Matsumura K \& Nakayama T. Effects of progesterone on thermosensitive neurons in preoptic slice preparations. Neuroscience Letters 198886 56-60. (https://doi.org/10.1016/0304-3940(88)90182-6)

43 Matsuda-Nakamura M, Yasuhara S \& Nagashima K. Effect of menstrual cycle on thermal perception and autonomic thermoregulatory responses during mild cold exposure. Journal of Physiological Sciences 201565 339-347. (https://doi.org/10.1007/ s12576-015-0371-x)

44 Rodriguez-Cuenca S, Monjo M, Gianotti M, Proenza AM \& Roca P. Expression of mitochondrial biogenesis-signaling factors in brown adipocytes is influenced specifically by 17beta-estradiol, testosterone, and progesterone. American Journal of Physiology: Endocrinology and Metabolism 2007292 E340-E346. (https://doi.org/10.1152/ ajpendo.00175.2006)

45 Lopez M \& Tena-Sempere M. Estradiol effects on hypothalamic AMPK and BAT thermogenesis: a gateway for obesity treatment? Pharmacology and Therapeutics 2017178 109-122. (https://doi. org/10.1016/j.pharmthera.2017.03.014)

46 Shorakae S, Jona E, de Courten B, Lambert GW, Lambert EA, Phillips SE, Clarke IJ, Teede HJ \& Henry BA. Brown adipose tissue thermogenesis in polycystic ovary syndrome. Clinical Endocrinology 201990 425-432. (https://doi.org/10.1111/cen.13913)

Received 2 March 2020

Revised version received 13 May 2020

Accepted 4 June 2020 